\title{
Ação acaricida in vitro de Tropaeolum majus sob teleóginas de Rhipicephalus (Boophilus) microplus
}

\author{
In vitro acaricidal effect of Tropaeolum majus on the engorged female of Rhipicephalus \\ (Boophilus) microplus
}

\section{Felipe Lamberti Pivoto ${ }^{I^{*}}$ Andréia Buzatti $^{\mathrm{I}}$ Felipe da Silva Krawczak ${ }^{\mathrm{I}}$ Giovana Camillo $^{\mathrm{I}}$ Luís Antônio Sangioni ${ }^{I}$ Gilberto Dolejal ZanettiI ${ }^{\text {II }}$ Melânia Palermo Manfron ${ }^{\mathrm{II}}$ Fernanda Silveira Flores Vogel ${ }^{\mathrm{I}}$}

\section{RESUMO}

O objetivo desse estudo foi o de avaliar a eficácia acaricida in vitro do extrato aquoso e etanólico a $70 \%$ da Tropaeolum majus em teleóginas do Rhipicephalus (Boophilus) microplus. Extrato das partes aéreas (caule e folhas) e de flores foram testados na concentração final de $2 \%$. A coleta de teleóginas de Rhipicephalus (Boophilus) microplus foi realizada em propriedades do município de São Francisco de Assis e São Pedro do Sul, Rio Grande do Sul, Brasil. O extrato das partes aéreas em etanol a $70 \%$ alcançou eficácia de 99,1\%. Esse estudo é o primeiro relato de ação carrapaticida de Tropaeolum majus.

Palavras-chave: Resistência, Rhipicephalus (Boophilus) microplus, Plantas medicinais, Tropaeolum majus, Tropaeolaceae, Capuchinha

\section{ABSTRACT}

The aim of this study was to evaluate the in vitro acaricide effect of aqueous and ethanolic extracts of Tropaeolum majus on Rhipicephalus (Boophilus) microplus engorged females. Extracts from the aerial parts (stems and leaves) and from the flowers were tested at a final concentration of 2\%. The Rhipicephalus (Boophilus) microplus engorged females were collected in farms in two municipalities (São Francisco de Assis and São Pedro do Sul), in Rio Grande do Sul state, Brazil. An efficacy of 99,1\% was found using extract of aerial parts in ethanol at 70\%. This is the first report of acaricidal effect of Tropaeolum majus.

Key words: Resistance, Rhipicephalus (Boophilus) microplus, Medicinal plants, Tropaeolum majus, Tropaeolaceae, Capuchinha.

\section{INTRODUÇÃO}

A infestação pelo carrapato Rhipicephalus (Boophilus) microplus vem provocando significativos prejuízos na bovinocultura, em áreas tropicais e subtropicais, causando um impacto econômico de dezenas de bilhões de dólares por ano, em todo o mundo (GASPARIN et al., 2007). Estes prejuízos são ocasionados principalmente pelo hematofagismo do parasita, que pode comprometer a produção de carne e leite, pela inoculação de toxinas nos hospedeiros, pelos custos diretos para seu controle e pela transmissão de agentes infecciosos (GHOSH et al., 2006).

O uso dos acaricidas constitui o principal instrumento de controle dos carrapatos, no entanto, atualmente, esse controle caracteriza-se pela crescente resistência parasitária e, conseqüentemente, aumento da frequência de aplicações desses produtos, podendo gerar prejuízos ao meio ambiente, ao homem e aos animais (ROCHA et al., 2006). No Brasil, a resistência de carrapatos $\boldsymbol{R}$. (B.) microplus a carrapaticidas foi verificada por diversos autores, dentre os quais PEREIRA (2006), na região do Vale do Paraíba, no estado de São Paulo; SILVA et al. (2000), em Goiânia; e CAMILLO et al. (2009) no estado do Rio Grande do Sul.

A utilização dos fitoterápicos no controle de carrapatos resulta em um desenvolvimento mais

'Laboratório de Doenças Parasitárias (LADOPAR), Departamento de Medicina Veterinária Preventiva (DMVP), Centro de Ciências Rurais (CCR), Universidade Federal de Santa Maria (UFSM). Prédio 44, Rua Roraima, 1000, 97105-000, Camobi. Santa Maria, RS, Brasil. E-mail: felipe.pivoto@gmail.com. *Autor para correspondência.

"Laboratório de Farmacognosia, Departamento de Farmácia Industrial (DFI), Centro de Ciências da Saúde (CCS), UFSM, Santa Maria, RS, Brasil. 
lento da resistência parasitária, além de apresentar características ecológicas desejáveis como: biodegradável, não causar poluição ambiental, diminuir drasticamente os resíduos no meio ambiente (CHAGAS, 2004) e uma melhor relação de custobenefício, representando redução na utilização de agroquímicos (AVANCINI, 1994; HEIMERDINGER et al., 2006).

Diferentes tipos de plantas com potencial fitoterápico foram descritas, sendo a estas atribuídas atividades anti-inflamatórias, bactericidas e acaricidas. Dentre essas, a Tropaeolum majus, popularmente conhecida como capuchinha, a qual foi observada atividade antimicrobiana (ZANETTI et al., 2003) e anticoagulante (SANTO, et al., 2007), ZANETTI et al. (2003) constataram que extratos aquosos e etanólicos a $70 \%$ das partes aéreas dessa planta não ocasionaram toxicidade aguda quando administrada in vivo em camundongos. Essa planta ocorre em centros de diversidade primária no sul do México até a Patagônia na Argentina e parte norte do Brasil (COMBA et al., 1999; PINTÃO et al., 1995), sendo que é cultivada pela rusticidade e facilidade de adaptação a climas variados (SANGALLI et al., 2004), tornando-se relevante a sua investigação, pela necessidade de estudos referentes a plantas medicinais domesticadas e de ocorrência no território nacional (ZANETTI, 2002).

O presente estudo teve por objetivo analisar a eficácia acaricida in vitro do extrato de $\boldsymbol{T}$. majus no controle de $\boldsymbol{R}$. (B.) microplus, demonstrando a eficácia da utilização de extratos desta planta medicinal na redução da freqüência do uso de acaricidas.

\section{MATERIALEMÉTODOS}

Obtenção e extração do material vegetal

O material vegetal constituiu-se de flores, folhas e caules de Tropaeolum majus coletado no município de Santa Maria, Rio Grande do Sul, Brasil, conforme exsicatas HDSI144 depositadas no herbário do Departamento de Botânica da Universidade Federal de Santa Maria.

As partes aéreas (caule e folha) e as flores de T. majus foram submetidas separadamente à desidratação e estabilização em estufa a $40 \pm 2^{\circ} \mathrm{C}$, moagem em macromoinho de facas e estocadas em frasco âmbar. Após, obteve-se o extrato bruto (EB) das partes aéreas e das flores, através da maceração em etanol a $70 \%$ com renovação de solvente. $\mathrm{O}$ extrato bruto foi reduzido a extrato seco (ES) por evaporação do solvente (SIMÕES et al., 2005).

Para realização dos ensaios in vitro o extrato seco foi retomado, parte em etanol a 70\% e outra parte em água destilada, ambos na concentração final de $2 \%$.
Coleta dos ácaros

Foram coletadas aproximadamente 300 teleóginas de $\boldsymbol{R}$. (B) microplus, com medidas entre 4,5 a 7,5mm de diâmetro, de bovinos mestiços, de ambos os sexos e de diferentes faixas etárias, naturalmente infestados. As coletas foram realizadas em duas propriedades, localizadas nos municípios de São Francisco de Assis ( $29^{\circ} 33^{\prime} \mathrm{S}$ e $55^{\circ} 07^{\prime} \mathrm{W}$, altitude $151 \mathrm{~m}$ ),

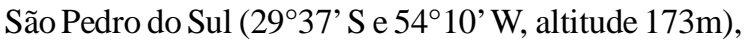
mesorregião sudoeste e mesorregião centro ocidental do estado do Rio Grande do Sul, respectivamente. Segundo os proprietários, o controle acaricida de $\boldsymbol{R}$. (B.) microplus utilizado nas duas propriedades durante os últimos anos era realizada com amitraz, através de banhos de aspersão e imersão, sendo que o amitraz apresentou-se eficaz e ineficaz, respectivamente, nas propriedades de São Francisco de Assis e São Pedro do Sul, através do teste de biocarrapaticidograma (Dados não demonstrados).

Foi respeitado um intervalo mínimo de 60 dias do último tratamento acaricida para realizar a coleta dos ácaros. As amostras coletadas foram devidamente identificadas, acondicionadas em caixas térmicas e transportadas até o Laboratório de Doenças Parasitárias da Universidade Federal de Santa Maria, onde permaneceram sob refrigeração a $4^{\circ} \mathrm{C}$, não excedendo o tempo de $48 \mathrm{~h}$ da coleta a realização dos testes in vitro.

Ensaio in vitro (biocarrapaticidograma)

A técnica de Biocarrapaticidograma foi realizada conforme DRUMMOND et al. (1973), em triplicata. Foram selecionadas 10 teleóginas por grupo com base na motilidade normal e aparência (corpo íntegro e máximo ingurgitamento) (LEITE et al., 1995), lavadas em água corrente, secas em papel absorvente, pesadas e separadas em seis grupos teste.

Os grupos foram submetidos a tratamentos como a seguir: (G1) Extrato das partes aéreas (caules e folhas) em etanol a 70\%; (G2) Extrato das partes aéreas em água destilada; (G3) Extrato da flor em água destilada; (G4) Solução etanólica a 70\%; (G5) Cipermetrina $8 \%$ e ethion $60 \%$; (G6) Controle água destilada.

Cada grupo foi submetido à imersão por cinco minutos, em béqueres contendo $20 \mathrm{ml}$ das respectivas soluções, sob constante agitação durante o período de imersão. A seguir, as teleóginas foram retiradas das soluções, secas em papel absorvente, e logo, fixadas através de fita dupla face em placas de Petri, sendo a seguir mantidas em estufa climatizada 
$\left(27^{\circ} \mathrm{C}\right.$ e UR $\left.80 \%\right)$ por 14 dias para a realização de ovoposição. Após este período foi realizada a avaliação do peso total de ovos de cada grupo, separando-se $0,3 \mathrm{~g}$ de ovos por grupo em tubo de ensaio, identificados individualmente, vedados com algodão hidrófilo, permanecendo por mais 26 dias, em estufa para a eclosão total e análise da eclodibilidade com estereomicroscópio. A eficácia dos extratos foi determinada de acordo com o cálculo da reprodução estimada, proposto por DRUMMOND et al. (1973).
$E R=\underline{\text { Peso da massa de ovos X \% eclosão X } 20000}$ Peso da massa de fêmeas
$\mathrm{ER}=$ Eficiência Reprodutiva
$\mathrm{EP}=\underline{\mathrm{ER} \text { do grupo controle }-\mathrm{ER} \text { do grupo tratado } \mathrm{X} 100}$ ER do grupo controle
$\mathrm{EP}=$ Eficiência do produto

Análise estatística

Os dados foram submetidos à análise estatística, utilizando-se de medidas de variabilidade para análise da ação na inibição da postura e a eficácia do extrato/produto. Considerando-se como eficácia o valor mínimo de $95 \%$, conforme legislação pertinente à comercialização de carrapaticidas no País (BRASIL, 1990). Quanto à eficiência reprodutiva das teleóginas e a eclodibilidade dos ovos, os resultados foram submetidos ao Teste de Tukey, com significância de $10 \%$ ( $\mathrm{P}<0,1)$, através do programa estatístico SAS (1997).

\section{RESULTADOS}

Os resultados referentes à inibição da postura e eficiência do extrato estão demonstrados na tabela 1. A ação do extrato das partes aéreas de T. majus em etanol a 70\% (G1) na inibição da postura apresentou valor médio de $50 \%$ e eficácia de $99,1 \%$. O G2 inibiu a postura em 13,3\% alcançando uma eficácia carrapaticida de $84,6 \%$. O G3, composto pelo extrato da flor em água destilada, atingiu inibição de postura média de 20,0\%, com eficácia de 63,5\%. O G4 (etanol a 70\%) apresentou eficácia de $33,0 \%$ e ação na inibição da postura nula. $\mathrm{O}$ controle positivo (G5) demonstrou 95\% de inibição da postura, alcançando $100 \%$ de eficácia.

Os valores referentes à eficiência reprodutiva dos diferentes grupos são demonstrados na tabela 1.

\section{DISCUSSÃO}

A T. majus vem sendo utilizada em estudos buscando analisar inúmeras aplicações medicinais (BLOEM et al., 2008; GASPAROTTO JR. et al., 2009; PINTÃO et al., 1995; SANTO et al., 2007; ZANETTI et al., 2003), porém resultados referentes à sua ação acaricida não foram realizados até o presente momento. Pesquisas demonstram a variação de resultados entre extratos de uma mesma planta, como pesquisado por COSTA et al. (2008), que ao utilizarem extratos hidroalcoólicos de citronela a 20\% (Cymbopogon nardus) verificaram que esse não apresentou eficácia acaricida (17\%). Contudo, os resultados de OLIVIO et al. (2008) demonstram que o óleo desta planta apresentou atividade acaricida sobre teleóginas de $\boldsymbol{R}$. (B.) microplus.

Foi possível verificar que os extratos testados de T. majus quando diluídos a $2 \%$ em etanol a $70 \%$ apresentaram eficácia satisfatória ( $>95 \%)$ segundo a legislação pertinente à comercialização de carrapaticidas no País (BRASIL, 1990), diferentemente de quando utilizado a água como solvente (Tabela 1), o que demonstra o efeito potencializador do álcool. Em estudo realizado com a planta Cymbopogon citratus, SILVA et al. (2008) observaram resultados semelhantes. A ação da T. majus em etanol a $70 \%$ foi mais efetiva no momento da eclosão dos ovos (Tabela 1). Ação semelhante foi registrada por PIRES et al. (2007) com o extrato das cascas de Simarouba versicolor, os quais verificaram que apesar de haver postura, os ovos das teleóginas não apresentaram

Tabela 1 - Ação do extrato de Tropaeo lum majus em diferen tes condições sobre Rhipicephalus (Boophilus) microplus.

\begin{tabular}{ccccc}
\hline Tratamento & $\begin{array}{c}\text { Grupo de teleóginas } \\
(\mathrm{n}=10)\end{array}$ & $\begin{array}{c}\text { Ação na inibição da } \\
\text { postura }(\%) \\
(\text { Média } \pm \text { Desvio Padrão) }\end{array}$ & $\begin{array}{c}\text { Eficiência reprodutiva } \\
\text { Tédia } \pm \text { Desvio Padrão) }\end{array}$ & $\begin{array}{c}\text { Eficácia do produto/extrato } \\
(\%)\end{array}$ \\
$\begin{array}{c}\text { Tukey } \\
\text { (Média } \pm \text { Desvio Padrão) }\end{array}$ \\
\hline G1 & $2,5 \pm 0,0$ & $50,0 \pm 40,0$ & $455631^{\mathrm{a}}$ & $99,1 \pm 1,0$ \\
G2 & $2,5 \pm 0,0$ & $13,3 \pm 5,7$ & $7961^{\mathrm{b}}$ & $84,6 \pm 2,0$ \\
G3 & $2,0 \pm 0,0$ & $20,0 \pm 28,2$ & $403011^{\mathrm{a}}$ & $63,5 \pm 12,0$ \\
G4 & $1,6 \pm 0$, & $0,0 \pm 0,0$ & $655967^{\mathrm{a}}$ & $33,0 \pm 29,6$ \\
G5 & $1,8 \pm 0,2$ & $95,0 \pm 7,0$ & $0^{\mathrm{c}}$ & $100 \pm 0,0$ \\
G6 & $1,9 \pm 0,1$ & $3,3 \pm 5,7$ & - & - \\
\hline
\end{tabular}

G1: Extrato das partes aéreas em etanol a 70\%; G2: Extrato das partes aéreas em água destilada; G3: Extrato das flores em água destilada; G4: Etanol a 70\%; G5: Controle químico positivo; G6: Control e água destilada.

Letras dis tintas, na coluna, indicam diferença significativa estatisticamente $(\mathrm{P}<0,1)$ pelo teste de Tukey. 
eclodibilidade, sendo entretanto diferente da ação de Citronela de Java (Cymbopogon winterianus Jowitt) a $10 \%$, para a qual não se verificou inibição na postura (MARTINS, 2006).

Em relação à ação dos extratos obtidos a partir da flor de T. majus observou-se que essa parte da planta apresenta metabólitos com ação acaricida, porém em níveis não satisfatórios, diferente dos resultados encontrados quando da utilização dos extratos das partes aéreas (caule e folhas) de T. majus (Tabela 1). Esse estudo corroborou com a análise realizada por CHAGAS (2004), o qual verificou oscilação significativa dos resultados, quando há disparidade em relação às partes, forma e época de colheita, estágio de desenvolvimento e forma de obtenção da planta a ser utilizada.

O etanol a 70\% (G4) não interferiu na postura das teleóginas (Tabela 1), porém exerceu um leve efeito sobre a eclodibilidade dos ovos de $\boldsymbol{R}$. (B.) microplus. Esse resultado é semelhante ao encontrado por SILVA et al. (2008) os quais observaram que o tratamento com álcool, reduziu a eclodibilidade, porém não interferiu na eficiência reprodutiva.

Os resultados da eficiência reprodutiva (Tabela 1) indicaram que os extratos das partes aéreas e das flores de T. majus em água destilada e o Etanol a $70 \%$ não demonstraram diferença significativa $(\mathrm{P}<0,1)$, mas quando comparados com o extrato das partes aéreas em etanol a 70\% apresentaram diferença significativa $(\mathrm{P}<0,1)$, assim como, a cipermetrina a $8 \% \mathrm{e}$ ethion a $60 \%$, apresentando entretanto diferença significativa $(\mathrm{P}<0,1)$ quando em comparação com os demais tratamentos. Isto se contrapõe aos resultados encontrados por MAGADUM et al. (2009), os quais em trabalhos utilizando as plantas Annona squamosa e Azadirachta indica não observaram diferença significativa $(\mathrm{P}<0,05)$ entre o controle positivo e os extratos utilizados, conferindo valores distintos $(\mathrm{P}<0,05)$ ao estudar oito concentrações do extrato de A. squamosa.

O produto acaricida a base de cipermetrina $8 \%$ e ethion $60 \%$ apresentou eficácia satisfatória (>95\%), vindo ao encontro dos resultados encontrados por CAMILLO et al. (2009), que também apresentou uma eficácia superior a 95\%, em $37 \%$ das propriedades testadas no estado do Rio Grande do Sul (Brasil). PEREIRA (2006), em estudo realizado com bovinos de leite no Vale do Paraíba, Estado de São Paulo, obtiveram na associação de cipermetrina $8 \%$ e ethion $60 \%$, resultados variando entre $59,34 \%$ e $100 \%$ de eficácia contra $\boldsymbol{R}$. (B.) microplus, evidenciando uma grande oscilação de resultados quando da utilização dessa combinação.

\section{CONCLUSÃO}

Os extratos de $\boldsymbol{T}$. majus testados apresentam ação acaricida in vitro na concentração de $2 \%$, oscilando sua eficácia conforme parte da planta testada e de seu diluente, sendo o extrato utilizado em etanol a $70 \%$ o mais eficiente frente a cepas de $\boldsymbol{R}$. (B.) microplus.

\section{REFERÊNCIAS}

AVANCINI, C.A.M. Sanidade animal na agroecologia atitudes ecológicas de sanidade animal e plantas medicinais em medicina veterinária. Porto Alegre: Fundação Gaia, 1994. p.33, 46p.

BLOEM, E. et al. Influence of Tropaeolum majus supplements on growth and antimicrobial capacity of glucotropaeolin in piglets. Landbauforschung Volkenrode, v.58, n.3, p.203210, 2008. Disponível em: <http://www.bfafh.de/bibl/lbf-pdf/ landbauforschung/vti-landbau forschung_58_3.pdf\#page $=63>$. Acesso em: 16 ago. 2010.

BRASIL, Ministério da Agricultura. Portaria n.90 de 04 de dez. de 1989. Normas para produção, controle e utilização de produtos antiparasitários. Diário Oficial, 22 janeiro de 1990, seção 1 , coluna 2 .

CAMILLO, $\mathrm{G}$ et al. Eficiência in vitro de acaricidas sobre carrapatos de bovinos no Estado do Rio Grande do Sul, Brasil. Ciência Rural, v.39, n.2, p.490-495, 2009. Disponível em: 〈http://www.scielo.br/pdf/cr/v39n2/a82cr262.pdf>. Acesso em: 12 ago. 2010. doi: 10.1590/S010384782008005000082 .

CHAGAS, A.C.S. Controle de parasitas utilizando extratos vegetais. Revista Brasileira de Parasitologia Veterinária, Supl.1, p.156-60, 2004

COMBA, L. et al. Garden flowers: insects visits and the floral reward horticulturally: modified variants. Annals of Botany Company, v.83, p.73-86, 1999. Disponível em: <http:// a ob.oxfordjournals.org/cgi/reprint/83/1/ $73 ? \mathrm{max}$ to show $=\&$ hits $=10 \&$ RES ULTFORMAT $=1$ \&andorexacttitle $=$ and \&andorexacttitleabs $=$ and \&andorexactfulltext= and \& se archid $=1 \&$ FIRST IN DEX $=0 \&$ sortspe $\mathrm{c}=\mathrm{re}$ levance $\&$ volume $=83 \&$ firstpage $=73 \&$ resourcetype $=$ HWCIT $>$. Acesso em: 12 ago. 2010. doi: 10.1006/anbo.1998.0798.

COSTA, F.B. et al. Eficácia de fitoterápicos em fêmeas ingurgitadas de Boophilus microplus, provenientes da mesorregião oeste do Maranhão, Brasil. Revista Brasileira de Parasitologia Veterinária, Supl.1, p.83-86, 2008. Disponível em: < http://cbpv.com.br/rbpv/documentos/ 17supl.12008/Artropode017.pdf>. Acesso em: 16 ago. 2010.

DRUMMOND, R.O. et al. Boophilus annulatus and Boophilus microplus: laboratory tests for insecticides. Journal of Economic Entomology, v.66, p.130-133, 1973. Disponível em: 〈http://www.ncbi.nlm.nih.gov/pubmed/4690254? $\log \$=$ activity> Acesso em: 12 ago. 2010.

GASPARIN, G. et al. Mapping of quantitative trait loci controlling tick Rhipicephalus (Boophilus) microplus resistance on bovine chromosomes 5, 7 and 14. Animal Genetics, v.37, p.1-7, 2007. Disponível em: < http://onlinelibrary.wiley.com/ doi/10.1111/j.1365-2052.2007.01634.x/pdf>. Acesso em: 12 ago. 2010. doi:10.1111/j.1365-2052.2007.01634.x. 
GASPAROTTO Jr., A. et al. Natriuretic and diuretic effects of Tropaeolum majus (Tropaeolaceae) in rats. Journal of Ethnopharmacology, v.122, n.3, p.517-522, 2009. Disponível em: <http:// www.sciencedirect.com/science?_ob=ArticleURL\&_udi=B6T8D4 VGF3 V $0-6 \&$ _ u ser $=687358 \&$ _ cover D a te $=04 \%$ $2 \mathrm{~F} 21 \% 2 \mathrm{~F} 2009 \& \_r d o c=1 \&$ fmt $=$ high\&_orig $=$ search\&_sort $=\mathrm{d} \&$ _ docanchor $=\&$ vie w $=$ c \&_a c c t $=$ C $000037899 \&$ _ version $=1 \&$ _ ur 1 Version $=0 \&$ _ userid $=68735 \overline{8}$ $\& m d 5=1304$ ccce9aefae035ad07e07ba79fcc0. Acesso em: 14 ago. 2010. doi: 10.1016/j.jep.2009.01.021.

GHOSH, S. et al. Control of ticks of ruminants with special emphasis on livestock farming system in India - present and future possibilities for integrated control: areview. Experimental and Applied Acarology, v.40, n.1, p.49-66, 2006. Disponível em: <http://www.springerlink.com/content/ 2542722q2h751020/>. Acesso em: 15 ago. 2010. doi: 10.1007/ s10493-006-9022-5.

HEIMERDINGER, A. et al. Extrato alcoólico de capim-cidreira no controle do Boophilus microplus em bovinos. Revista Brasileira de Parasitologia Veterinária, v.15, n.1, p.3739, 2006. Disponível em: <http://cbpv.com.br/rbpv/ documentos/1512006/c15137_39.pdf>. Acesso em: 15 ago. 2010 .

LEITE, R.C. et al. Efficacy of doramectin against natural infestations of Boophilus microplus (Canestrini, 1887) (Acari: Ixodidade) in cattle. Revista Brasileira de Parasitologia Veterinária, v.4, n.1, p.53-56, 1995. Disponível em: <http:/ /cbpv.com.br/rbpv/documentos/411995/c4153_56.pdf>. Acesso em: 18 ago. 2010.

MAGADUM, S. et al. Comparative efficacy of Annona squamosa and Azadirachta indica extracts against Boophilus microplus Izatnagar isolate. Parasitology Research, v.105, n.4, p.1085-1091, 2009. Disponível em: <http:// www.springerlink.com/content/a625501 $78504105 />$. Acesso em: 12 ago. 2010. doi: 10.1007/s00436-009-1529-3.

MARTINS, R.M. Estudio in vitro de la acción acaricida del aceite esencial de la gramínea Citronela de Java (Cymbopogon winterianus Jowitt) en la garrapata Boophilus microplus. Revista Brasileira de Plantas Medicinais, v.8, n.2, p.7178, 2006. Disponível em: <http://www.ibb.unesp.br/servicos/ publicacoes/rbpm/pdf_v8_n2_2006/artigo12_v8_n2.pdf >. Acesso em: 13 ago. 2010.

OLIVIO, C.J. et al. Óleo de citronela no controle do carrapato de bovinos. Ciência Rural, v.38, n.2, p.406410, 2008. Disponível em: < http://www.scielo.br/ scielo.php? script $=$ sci_arttext \& pid $=$ S $0103-$ $84782008000200018 \& \operatorname{lng}=\mathrm{pt} \& \mathrm{nrm}=\mathrm{iso} \& \mathrm{t} \operatorname{lng}=\mathrm{pt}>$. Acesso em; 14 ago. 2010. doi: 10.1590/s0103-84782008000200018.

PEREIRA J.P. Eficácia in vitro de formulações comerciais de carrapaticidas em teleóginas de Boophilus microplus coletadas de bovinos leiteiros do Vale do Paraíba, estado de São Paulo. Revista Brasileira de Parasitologia Veterinária, v.15, n.2, p.45-48, 2006. Disponível em: < http://cbpv.com.br/rbpv/ documentos/1522006/c15245_48.pdf>. Acesso em: 13 ago. 2010 .

PINTÃO, A.M. et al. In vitro antitumor activity of benzyl isothyocianate: a natural product from Tropaeolum majus. Planta Medica, v.61, p.233-236, 1995. Disponível em: < http://www.ncbi.nlm.nih.gov/pubmed>. Acesso em: 18 ago. 2010 .
PIRES, J.E.P. et al. Determinação da concentração inibitória média (CI50) do extrato aquoso de Simarouba versicolor, St. Hill sobre a ovipostura do carrapato bovino (Boophilus microplus, Canestrine, 1887). Revista Brasileira de Plantas Medicinais, v.9, n.4, p.23-26, 2007. Disponível em: <http:/ /www.ibb.unesp.br/servicos/publicacoes/rbpm/pdf_v9_n4_2007/ artigo4_v9_n4.pdf>. Acesso em: 12 ago. 2010.

ROCHA, C.M.B.M. et al. Percepção dos produtores de leite do município de Passos, MG sobre o carrapato Boophilus microplus (Acari:Ixodidae), 2001. Ciência Rural, v.36, n.4, p.1235-1242, 2006. Disponível em: < http://www.scielo.br/ pdf/cr/v36n4/a29v36n4.pdf>. Acesso em: 14 ago. 2010.

SANGALLI, A. et al. Resíduos orgânicos e nitrogênio na produção de biomassa de capuchinha (Tropaeolum majus $L$.) Jewel. Revista Ciência e Agrotecnologia, v.28, n.4, p.831839, 2004. Disponível em: < http://www.editora.ufla.br/revista/ 28_4/art15.PDF>. Acesso em: 12 ago. 2010.

SAS, Statistical Analisys System. User's guide Stat. 2.ed. Cary, 1997. 456p. Disponível em: <http://www.umass.edu/ $\mathrm{statdata/s} \mathrm{of} \mathrm{tware/new} \mathrm{s/s} \mathrm{p} \mathrm{s} \mathrm{s/manua} 1 \mathrm{~s} 18 /$ PASW\%20Statistics\%2018\%20Core\%20System\%20User's\%20Guide.pdf $>$. Acesso em: 18 ago. 2009.

SANTO, A.P.E. et al. Anticoagulant in vitro effect of hidrotethanolic extract of edible leaves and flowers of Tropaeolum majus L. (Tropaeolaceae) on human plasma. Latin American Journal of Pharmacy, v.23, n.5, p.732736, 2007. Disponível em: < http://www.latamjpharm.org/ trabajos/26/5/LAJOP_26_5_2_1_27XD126D18.pdf>. Acesso em: 18 ago. 2010.

SILVA, F.F. et al. Avaliação comparativa da eficácia de fitoterápicos e produtos químicos carrapaticidas no controle do Boophilus microplus (Canestrini, 1887) por meio do biocarrapaticidograma. Revista Medicina Veterinária do Departamento de Medicina Veterinária da Universidade Federal Rural de Pernambuco, v.2, n.3, p.1-8, 2008. Disponível em: < http://www.dmv.ufrpe.br/revista/ Avaliacao_comparativa_v2n3.pdf $>$. Acesso em: 13 ago. 2010.

SILVA, M.C.L. et al. Avaliação in vitro da eficácia do Clorfenvinfós e da Cialotrina sobre o Boophilus microplus colhidos em bovinos da bacia leiteira da microrregião de Goiânia, Goiás. Ciência Animal Brasileira, v.1, n.2, p.143-148, 2000. Disponível em: < http://www.revistas.ufg.br/index.php/ vet/article/view/244/216>. Acesso em: 14 ago. 2010.

SIMÕES C.M.O. et al. Farmacognosia da planta ao medicamento. 5.ed. Porto Alegre/Florianópolis: UFRGS/ UFSC, 2005. $821 \mathrm{p}$.

ZANETTI, G.D. Troapeolum majus L.: morfohistologia, fitoquímica, ação antimicrobiana e toxicidade. 2002. 90f. Dissertação (Mestrado em Ciência e tecnologia farmacêutica) - Universidade Federal de Santa Maria, Santa Maria, RS.

ZANETTI, G.D. et al. Toxicidade aguda e atividade antibacteriana dos extratos de Tropaeolum majus L. Acta Farmaceutica Bonaerense, v.22, n.2, p.159-162, 2003. 